\title{
An Integrated Artificial Potential Field Path Planning with Kinematic Control for Nonholonomic Mobile Robot
}

\author{
Hendri Himawan Triharminto ${ }^{\# *}$, Oyas Wahyunggoro ${ }^{\#}$ Teguh Bharata Adji ${ }^{\#}$, Adha Imam Cahyadi ${ }^{\#}$ \\ \# Electrical Engineering and Information Technology Department, Gadjah Mada University, Yogyakarta, Indonesia \\ E-mail:kanghimawan@gmail.com,oyas@ugm.ac.id; adji@mti.ugm.ac.id; masimam@jteti.gadjahmada.edu \\ * Electronic Department, Indonesia Air Force Academy, Yogyakarta, Indonesia
}

\begin{abstract}
In this paper, path planning which is based on Artificial Potential Field (APF) and the kinematic based control is integrated in order to solve an issue in the APF. Usually, the APF assumes the robot is modeled as a point mass. It means that the robot can move in any direction and neglect the nonholonomic constraint. In order to solve such a problem, the APF should be considered as part of the control system. This research proposed an approach integrating APF and control system under nonholonomic constraint. Naturally, the force of the APF can be used as linear velocity in the control system. Then, waypoint of APF is used as equilibrium point of kinematic control. In order to validate the proposed method, the experimental setup conducted on loop simulation. The scenario is that the robot moves along the certain trajectory to reach the goal point. The obstacle was set in between the robot and the goal point. The initial, goal, and the obstacles are set randomly. The experiments show that the integration of the proposed method can be implemented successfully. The real obstacle avoidance method and fulfilling the nonholonomic constraint are the proof that the method is running well. The results show that the integrated proposed method meets convergent and stable.
\end{abstract}

Keywords - APF; kinematic control; nonholonomic

\section{INTRODUCTION}

Based on the human loop, a robot can be divided into robot human interference and autonomous robot. Robot human interference means that the robot is controlled by human or human in the loop. An autonomous robot does not need a human loop in the operation. The research field of an autonomous robot growth is very extensive recently. The autonomous robot system is built by path planning, control system, and mission planning. The system of the autonomous robot can be illustrated in Fig. 1 .

Mission planning is a system which decides mission objective and goal position. Usually, planning system is higher level part than the control system [1][2]. The path planning is to guide the control system regarding to robot's movement [3]. With the guidance of the path planning system, the control system will move the actuator to reach the goal from the path planning system. In the path planning system, information about the environment from acquisition data sensor is obtained and used as data input.
From the Fig. 1, the process is a close-loop process until the mission planning and task allocation has been achieved. It has been done in many works that most of the path planning are assumed the robot as a point mass. It means that the robot can move in any direction and the path planning neglects kinematic constraints.

On the other hands, Artificial Potential Field (APF) is the most viable approach to build path planning for real time obstacle avoidance. Since the APF assumes the robot as a point mass, then it has to be modified regarding to kinematic constraint. This approach is purposed to compensate with the kinematic control system.

This research focuses on integrating the APF with feedback control system. The integration has applied the value of natural force of APF as velocity and discrete waypoint as equilibrium point of the control system. The description of the proposed method will be delivered in Section III. Formerly, the background of the study explained in Section II. The description is followed by the result and analysis in Section IV. The conclusion including the future work will be defined in the last Chapter. 


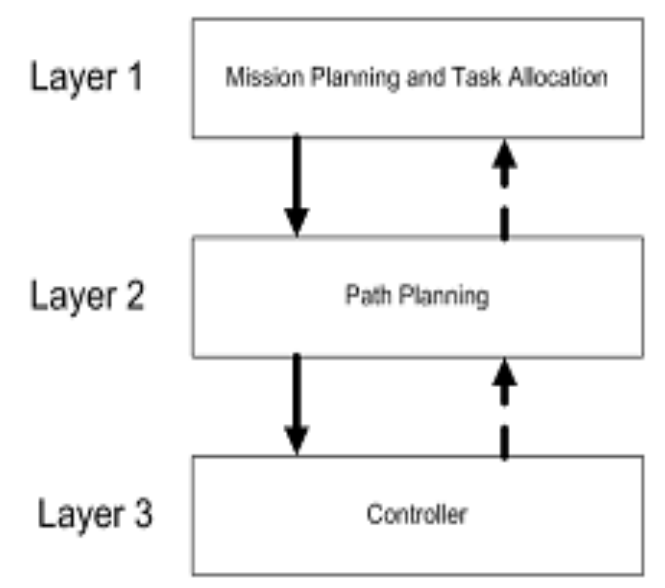

Fig. 1 System Layer of Autonomous Robot

\section{RELATED WORKS}

Initially, the major problem of robot generation was divided into two ways, i.e. considered as a control problem of a nonholonomic system and as a planning problem of a kinematic constraint [4].

Some evolutionary algorithms were introduced such as Genetic Algorithm (GA), Particle Swarm Optimization (PSO), Ant Colony Algorithm (ACA), Artificial Bee Colony (ABC), and Bacterial Evolutionary Algorithm (BEA) to solve planning problem. The aim of the path planning algorithm is to convert a mission planning into a reference trajectory which the robot is required to follow [5].

Some researchers used basic GA in the path planning problem [6][7]. In [8] the GA approach was used to generate a feasible trajectory for a mobile robot that satisfies a the nonlinear evolution. The idea of GA is also used in the path planning using BEA which more adaptive than GA [9][10][11]. The other evolutionary algorithms that were used to produce path planning were PSO [11] [12], [14], [15]. Each particle represents a potential solution that is evaluated by three factors: position, velocity, and adaptability. The adaptability means the trajectory satisfied the kinematic and dynamic control. Similar to PSO, ACA and ABC approach used ant and bee as the candidate solution [16], [17], [18]. The solution found the shortest path but neglected the kinematic constraint. Portas et al. found that Differential Evolution (DE) has better performance than the others evolutionary algorithm in the multi-objective criteria [19].

Evolutionary algorithm based techniques can generate a trajectory but neglected the generation of the control signal to actualize it.

In [20] $A^{*}$ algorithm is combined with APF algorithm to enhance the quality of the selected trajectories. The $A^{*}$ algorithm was used as path planning generator. The APF itself was responsible for obstacle avoidance during the actual trajectory. A probabilistic method such as RRT (Random Rapidly Tree) was used for manoeuvre planning in nonholonomic robots [5]. Similar to AI-based approach, A* with APF and RRT still distinguished path planning from the control system.

The curve algorithm is a path planner that considering kinematic constraint [21], [22], [23]. Dynamic path using curve introduced by [24]. The kinematic constraints are maximum curvatures and maximum torsion bound. Curve algorithm is used by some researchers to solve path planning with control system problem. In [25], Dubins curve and control Lyapunov function are combined to lay a trajectory for a mobile robot via these potential forces. Another Dubins curves combined with sliding mode control is employed by [26]. The assumption of robot like car is in constant linear velocity and manipulating angular velocity is the objective of the controller. The motion terminates at the signal's unique extremum.

In the control problem, the issue of guidance is not considered [4]. The goal point is provided to the robot as equilibrium point of the control system. The reference trajectory converts into control signal. A controller using a constant tangential velocity and an angular velocity that constructed from a sum of sinusoids generates the motion of a unicycle [27]. The harmonic potential field (HPF) approach can be used in the control system [28]. The HPF generates a feasible trajectory in the cluttered environment. The derivation of the trajectory is used as control signal to the robot. Similar to [28], the vector field orientation used as control signal of angular and linear velocities [29]. The signal controls the velocity of the robot based on the agreement with the reference velocity of the gradient field.

The potential field method is a real time obstacle avoidance method without a prior model of the obstacle [30]. The algorithm generates a potential function on the obstacle that drags the robot from the obstacle and generating the safety path [31]. An artificial potential function is constructed according to obstacles. Some of the researches had been modified the original APF [9],[32],[33]. All of the research based on evolutionary computation to optimize the APF parameters [34].

However, when the potential field method is applied to nonholonomic systems, the path could be infeasible [35]. It happens because the systems assumed the robot as point mass and there are an infinite number of points where the gradient vector is perpendicular to input vector fields. The impact is that the robot cannot always move along the gradient vector of the potential function compensating the nonholonomic constraints. Urakubo proposed feedback stabilization controller based on potential function [35], [36]. The input is derived from the gradient vector with an additional component that does not decrease and increase the potential function.

Hence, this research focuses on kinematic control system with the reference signal and trajectory by using the artificial potential field which can avoid the obstacle and reach convergent.

\section{THE PROPOSED METHOD}

In this research the assumption of the robot as a point mass is eliminated, then not only position in the absolute coordinate has to be considered but the direction of the robot must be included. The kinematic control referred on [37] is to compensate the nonholonomic constraint with APF as the reference of the path. The diagram process can be seen in Fig. 2.

The system above starts with APF employment which the input data is from the environment. The environment here is an obstacle, initial and goal position. The APF yield a path 
planning that can avoid the obstacle that is used as reference orientation of the kinematic control. Besides as reference orientation, the APF will use as part of control system. The control system uses two inputs, i.e. linear and angular velocities. From the Fig. 2, it can be shown that if the goal position is global optimum then the system will be terminated. Consequently, if the goal position is not the global solution then the processes will loop to the APF to calculate the next point.

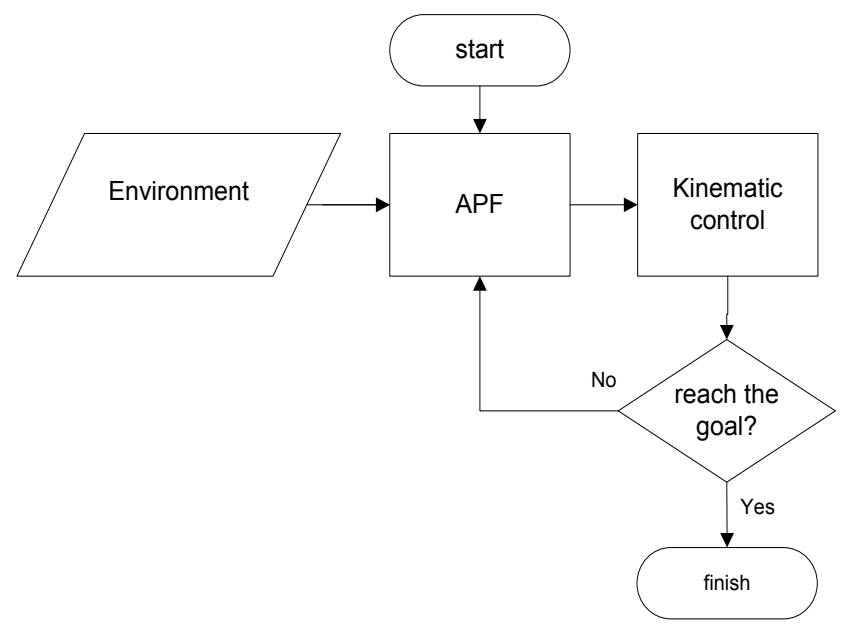

Fig. 2 Processes of the system

\section{A. Kinematic Control}

The kinematic of a differential-drive mobile robot in the inertial frame $\mathrm{X}_{\mathrm{I}}, \mathrm{Y}_{\mathrm{I}}$, and orientation $\theta$ is given by

$$
\left[\begin{array}{l}
\dot{x} \\
\dot{y} \\
\dot{\theta}
\end{array}\right]=\left[\begin{array}{cc}
\cos \theta & 0 \\
\sin \theta & 0 \\
0 & 1
\end{array}\right]\left[\begin{array}{l}
v \\
\omega
\end{array}\right],
$$

where $\dot{x}$ and $\dot{y}$ are the linear velocities in the direction of the $\mathrm{X}_{\mathrm{I}}$ and $\mathrm{Y}_{\mathrm{I}}$ of the initial frame. The $\theta$ variable is the angle between robot's heading (body frame) against the inertial frame. The control input is symbolized by $v$ and $\omega$. Notation $\omega$ is control input to generate the angular velocity and notation $v$ is to produce the linear velocity. The nonholonomic constraint is formulated by

$$
\dot{x} \sin \theta-\dot{y} \cos \theta=0
$$

that shows no lateral motion is allowed.

From the Fig. $3, \mathrm{X}_{\mathrm{R}}$ and $\mathrm{Y}_{\mathrm{R}}$ are body frame of the robot. Notation $\alpha$ is the angle between the $\mathrm{X}_{\mathrm{R}}$ axis of the robot body frame and the vector $x$ connecting the center of the axle of the wheels with the final position. It is assumed that the angle $\alpha$ has range from $-\pi / 2$ until $\pi / 2$. The kinematic model can be illustrated in Figure 3.

Figure 3 show that variable $\theta$ is the angle between the inertial frame and the body frame. Variable $\beta$ determines the angle of the robot and the origin point in the inertial frame. The angle has opposite direction against sum of $\alpha$ and $\theta$. In order to compensate the direction of the body frame, the coordinate is transformed into polar coordinates with its origin at the goal position

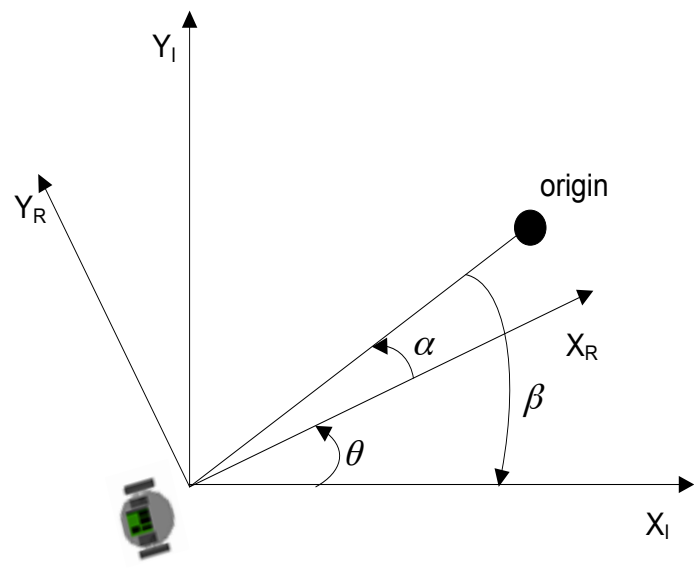

Fig. 3 Kinematic Model of Nonholonomic Robot

$$
\begin{aligned}
& \rho=\sqrt{\Delta x^{2}+\Delta y^{2}} \\
& \alpha=-\theta+a \tan 2(\Delta y, \Delta x) \\
& \beta=-\theta-\alpha .
\end{aligned}
$$

The new polar coordinates can be written in the following matrix equation.

$$
\left[\begin{array}{c}
\dot{\rho} \\
\dot{\alpha} \\
\dot{\beta}
\end{array}\right]=\left[\begin{array}{cr}
-\cos \alpha & 0 \\
\frac{\sin \alpha}{\rho} & -1 \\
\frac{-\sin \alpha}{\rho} & 1
\end{array}\right]\left[\begin{array}{l}
v \\
\omega
\end{array}\right],
$$

where $\rho$ as in (3) is the distance between the center of the robot's wheel axle and the goal position, $\theta$ is the angle between the $X_{R}$ axis of the robot and the reference frame, and the $\mathrm{X}_{\mathrm{I}}$ axis associated with the final position $v$ and $\omega$. The control signal $v$ and $\omega$ are used to drive the robot from its actual configuration. The linear and angular control laws are formulated as below.

$$
\begin{gathered}
v=k_{\rho} \rho \\
\omega=k_{\alpha} \alpha+k_{\beta} \beta
\end{gathered}
$$

Notation $v$ and $\omega$ in (6) can be replaced by (7) and (8). Then, a closed-loop system is described as

$$
\left[\begin{array}{c}
\dot{\rho} \\
\dot{\alpha} \\
\dot{\beta}
\end{array}\right]=\left[\begin{array}{l}
-k_{\rho} \rho \cos \alpha \\
k_{\rho} \rho \sin \alpha-k_{\alpha} \alpha-k_{\beta} \beta \\
-k_{\rho} \rho \sin \alpha
\end{array}\right]
$$


The close-loop system with $v>0$ explains that the robot moves in forward direction. It has to be noted that $k$ is the control parameters. From [37], the close-loop system is stable if

$$
k_{\rho}>0, k_{\alpha}-k_{\rho}>0, k_{\beta}<0 .
$$

\section{B. Artificial Potential Field}

APF is a simple method for constructing the path which follows the natural of potential field [38]. The idea that potential field moved from the highest to the lowest potential implied to the robot initial position and goal position. The goal position definitely becomes the lowest and the initial is representative of the highest potential. This potential in a certain position can be reflected as attractive force which can be modelled as

$$
f_{a}(x, y)=\nabla V_{a}(x, y)
$$

which $V_{a}(x, y)$ usually set as

$$
\frac{1}{2} K_{a}\left[\left(x-x_{T}\right)^{2}+\left(y-y_{T}\right)^{2}\right] \text {. }
$$

$K_{a}$ is the gain parameter and $\left(x_{T}, y_{T}\right)$ is the goal position.

As the real time of obstacle avoidance algorithm, APF has its own mechanism by building another potential field in the obstacle which is called repulsive. The mathematical model of the potential surface in a certain position is

$$
V_{o}(x, y)=\frac{K_{o}}{\sqrt{\left(x-x_{o}\right)^{2}+\left(y-y_{o}\right)^{2}}}
$$

and the repulsive APF is

$$
f_{x o}(x, y)=-\frac{\partial V_{o}(x, y)}{\partial x}
$$

and

$$
f_{y o}(x, y)=-\frac{\partial V_{o}(x, y)}{\partial y} .
$$

The total of the force field which determines the obstacle avoidance path in the real time is

$$
\begin{aligned}
& f_{x}(x, y)=f_{x a}(x, y)+f_{x o}(x, y) \\
& f_{y}(x, y)=f_{y a}(x, y)+f_{y o}(x, y) .
\end{aligned}
$$

Equation (16) means that the total force is sum of attractive and repulsive forces in the $\mathrm{x}$ axis. Equation (17) is total force of attractive and repulsive in the $y$ axis.

\section{Design of Artificial Potential Field Control}

The artificial potential field takes role on the kinematic control especially in (7) and (8). The APF method yields a transition position from the current position.

$$
r_{\text {pose }}\left(x_{t}, y_{t}\right)=r_{\text {pose }}\left(x_{t-1}, y_{t-1}\right)+f\left(x_{t}, y_{t}\right) \text {, }
$$

where $r_{\text {pose }}\left(x_{t}, y_{t}\right) \in \mathfrak{R}^{2}$ is the current position of the robot and $f\left(x_{t}, y_{t}\right)$ is the total of force field in a certain position. Notation $t$ is time domain. The direction can be obtained by using the robot pose as seen in Fig. 4.

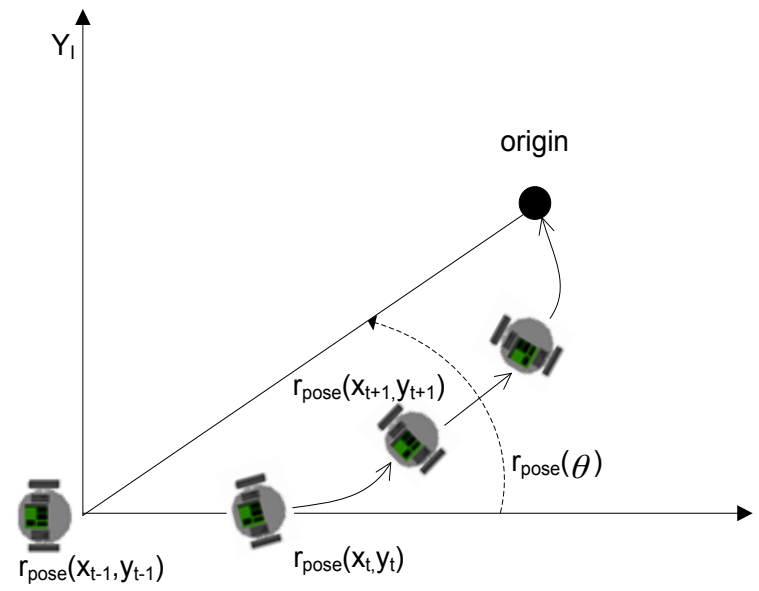

Fig. 4 APF Model of Nonholonomic Robot

The mathematical model is

$$
\underset{\substack{\text { pose } \\(19)}}{r}(\theta)=\arctan \left|\frac{r_{\text {pose }}\left(y_{t}\right)-\operatorname{goal}(y)}{r_{\text {pose }}\left(x_{t}\right)-\operatorname{goal}(x)}\right|
$$

Since the APF can give a direction and generate a force, it can be used as the equilibrium point in the control system. Thus, the idea of the proposed method is passing the states $r_{\text {pose }}\left(x_{t}, y_{t}\right)$ of APF to the kinematic control equilibrium point $(\dot{x}, \dot{y})$. The matrix formulation is given by

$$
\left[\begin{array}{l}
\dot{x} \\
\dot{y}
\end{array}\right]=\left[\begin{array}{c}
r_{\text {pose }}\left(x_{t}\right) \\
r_{\text {pose }}\left(y_{t}\right)
\end{array}\right] \text {. }
$$

From the (6), the design of the kinematic control considers direction and total force of the APF will influence to the control signal $v$ and $\omega$. From (3) and (7), the formula is nearly the same with (12). The formulas consider the distance to the origin or goal position. Therefore, control input $v$ can be replaced by total force magnitude of the APF in the certain time. The APF also decreases the velocity monotonically then it is appropriate for the control system. The control input $v$ is given by

$$
v=k_{\rho}\left\|f\left(x_{t}, y_{t}\right)\right\| \text {. }
$$


It has to be considered that the $f\left(x_{t}, y_{t}\right)$ sometimes obtains too small, then $k_{\rho}$ is a gain parameter to reach the minimum velocity which determines as small positive constant value. Magnitude $f\left(x_{t}, y_{t}\right)$ is obtained from attractive and repulsive force. The attractive forces are

$$
\begin{aligned}
& f_{x a}(x, y)=-K_{a}\left|x_{t}-x_{T}\right|, \\
& f_{y a}(x, y)=-K_{a}\left|y_{t}-y_{T}\right| .
\end{aligned}
$$

If the origin $\left(x_{T}, y_{T}\right)=(0,0)$ then

$$
\begin{aligned}
& f_{x a}(x, y)=-K_{a}\left|x_{t}\right|, \\
& f_{y a}(x, y)=-K_{a}\left|y_{t}\right| .
\end{aligned}
$$

The repulsive force with $\left(x_{T}, y_{T}\right)=(0,0)$ are

$$
\begin{aligned}
& f_{x o}=\frac{-K_{o}\left|x_{t}-o_{x}\right|}{\sqrt{\left[\left(x_{t}-o_{x}\right)^{2}+\left(y_{t}-o_{y}\right)^{2}\right]^{3}}}, \\
& f_{y o}=\frac{-K_{o}\left|y_{t}-o_{y}\right|}{\sqrt{\left[\left(x_{t}-o_{x}\right)^{2}+\left(y_{t}-o_{y}\right)^{2}\right]^{3}}}
\end{aligned}
$$

Then, the total of the force with $\left(x_{T}, y_{T}\right)=(0,0)$ in $\mathrm{x}$ axis is

$$
\begin{aligned}
f\left(x_{t}\right) & =f_{x a}+f_{x o} \\
& =-K_{a}\left|x_{t}\right|+\frac{K_{o}\left|x_{t}-x_{0}\right|}{\sqrt{\left[\left(x_{t}-o_{x}\right)^{2}+\left(y_{t}-o_{y}\right)^{2}\right]^{3}}}
\end{aligned}
$$

and the total of force in $y$ axis is

$$
\begin{aligned}
f\left(y_{t}\right) & =f_{y a}+f_{y o} \\
& =-K_{a}\left|y_{t}\right|+\frac{K_{o}\left|y_{t}-y_{o}\right|}{\sqrt{\left[\left(x_{t}-o_{x}\right)^{2}+\left(y_{t}-o_{y}\right)^{2}\right]^{3}}} .
\end{aligned}
$$

It has to be noted that the negative value in the (28)(29) due to the opposite direction of the force. It is assumed that the potential field force toward the point has a positive value and vice versa.

The total of attractive and repulsive forces is

$$
f\left(x_{t}, y_{t}\right)=
$$

$$
=\sqrt{f\left(x_{t}\right)^{2}+f\left(y_{t}\right)^{2}+2 f\left(x_{t}\right) f\left(y_{t}\right) \cos (\phi)},
$$

where

$$
\phi=a \tan \left(f\left(y_{t}\right) / f\left(x_{t}\right)\right),
$$

Thus, $v$ in (7) becomes

$$
v_{a p f}=k_{\rho} \sqrt{f\left(x_{t}\right)+f\left(y_{t}\right)+2 f\left(x_{t}\right) f\left(y_{t}\right) \cos (\phi)} .
$$

On the other hands, the angular velocity aims to control the direction of the robot. Equation (4) and (5) is the variable for the angular velocity. Variable $\alpha$ guides the robot to the origin. Considering APF as the real time obstacle avoidance, the angular velocity direction has to follow the safety path avoiding the obstacle. Consequently, the direction of variable $\alpha$ can be modified as

$$
\alpha_{a p f}=-\theta+a \tan 2\left(f\left(y_{t}\right), f\left(x_{t}\right)\right) .
$$

Putting $\alpha$ into $\beta$ becomes

$$
\begin{gathered}
\beta_{\text {apf }}=-\theta-\left(-\theta+a \tan 2\left(f\left(y_{t}\right), f\left(x_{t}\right)\right)\right. \\
=-a \tan 2\left(f\left(y_{t}\right), f\left(x_{t}\right)\right) .
\end{gathered}
$$

Using (6),(8),(32),(33), and (34), the closed-loop system can be written

$$
\left[\begin{array}{c}
\dot{\rho} \\
\dot{\alpha} \\
\dot{\beta}
\end{array}\right]=\left[\begin{array}{l}
-V_{a p f} \cos \alpha \\
k_{\rho} \rho \sin \alpha_{a p f}-k_{\alpha} \alpha_{a p f}+k_{\beta} \beta_{a p f} \\
-k_{\rho} \rho \sin \alpha_{a p f}
\end{array}\right] .
$$

In (33), the variable $\alpha$ purposes to go to the direction of the next position of the APF. It means that the direction avoids the obstacle in front. The modified of $\alpha$ will influence to $\beta$ and $\omega$ respectively.

\section{Stability Analysis of Artificial Potential Field Control}

If (32) compares with (7), then variable $\rho$ can be replaced with (30). Let assumes that the new variables are $\rho_{\text {apf }}, \alpha_{\text {apf }}$, and $\beta$ apf. Therefore, the close-loop system becomes

$$
\left[\begin{array}{l}
\dot{\rho}_{a p f} \\
\dot{\alpha}_{a p f} \\
\dot{\beta}_{a p f}
\end{array}\right]=\left[\begin{array}{l}
-k_{\rho} \rho_{a p f} \cos \alpha \\
k_{\rho} \rho_{a p f} \sin \alpha_{a p f}-k_{\alpha} \alpha_{a p f}+k_{\beta} \beta_{a p f} \\
-k_{\rho} \rho_{a p f} \sin \alpha_{a p f}
\end{array}\right]
$$

From [37], the stability of the system had been proven clearly. The linearization of (36) around the equilibrium $\left(\mathrm{x}_{\mathrm{T}}, \mathrm{y}_{\mathrm{T}}\right)=(0,0)$ which means $\cos \mathrm{x}=1$ and $\sin \mathrm{x}=\mathrm{x}$ can be written as

$$
\left[\begin{array}{l}
\dot{\rho}_{\text {apf }} \\
\dot{\alpha}_{\text {apf }} \\
\dot{\beta}_{\text {apf }}
\end{array}\right]=\left[\begin{array}{ccc}
-k_{\rho} & 0 & 0 \\
0 & -\left(k_{\alpha}-k_{\rho}\right) & -k_{\beta} \\
0 & -k_{\rho} & 0
\end{array}\right]\left[\begin{array}{l}
\rho_{\text {apf }} \\
\alpha_{\text {apf }} \\
\beta_{\text {apf }}
\end{array}\right] .
$$

The system is stable if the Eigen values of the matrix all have a negative real part. Let assumes that $\mathrm{A}$ is the matrix

$$
A=\left[\begin{array}{ccc}
-k_{\rho} & 0 & 0 \\
0 & -\left(k_{\alpha}-k_{\rho}\right) & -k_{\beta} \\
0 & -k_{\rho} & 0
\end{array}\right]
$$




$$
\left[\begin{array}{ccc}
\lambda+k_{\rho} & 0 & 0 \\
0 & \lambda+\left(k_{\alpha}-k_{\rho}\right) & 0 \\
0 & 0 & \lambda
\end{array}\right]
$$

The requirement of roots have negative real part if

$$
k_{\rho}>0 ; \quad-k_{\beta}>0 ; \quad k_{\alpha}-k_{\beta}>0
$$

In order to prove that the dynamic constraint is considered, the total force in the equilibrium point must equal to 0 .

1) Preposition 1. The kinematic control can be unstable in the origin (goal position) if the repulsive still applied on the control system and the obstacle is not in the origin. The obstacle assumes as a single point. The unstable condition can be modeled as

$$
x_{o} \neq x_{T} \text { and } y_{o} \neq y_{T} .
$$

\section{Proof of Preposition 1}

Let assumes that the origin is $(0,0)$. Then, the component of $f_{x a}$ and $f_{y a}$ will be equal to zero, but the component of $f_{x o}$ and $f_{y o}$ has a value and not equal to zero. Consequently, the control input $v$ generates signal input and the system is not convergent.

In order to solve the problem, this research delivered two conditions of control design that are explained in the following lines.

$$
f\left(x_{t}, y_{t}\right)=\left(f_{x a}, f_{y a}\right) \text { if } \rho_{o}>c_{o b s},
$$

where $\rho_{o}$ is distance between the robot against the obstacle and $c_{o b s}$ is the safety distance between the robot against the obstacle. The $c_{o b s}$ must fulfil that $c_{o b s}>0$.
2) Preposition 2. From (38), if the condition $\rho_{o}<c_{o b s}$ is reached, then the repulsive potential field part of the control system is not employed. Since the repulsive is not included, the local stability can be proven easily.

\section{Proof 1 of preposition 2}

Let assumes that point $\left(x_{T}, y_{T}\right)$ is $(0,0)$. If

$$
v_{a p f}=k_{\rho} \sqrt{f_{x a}^{2}+f_{y a}^{2}+2 f_{x a} f_{y a} \cos (\phi)},
$$

then $\left(\mathrm{x}_{\mathrm{t}}-\mathrm{x}_{\mathrm{T}}\right)=0$ and $\left(\mathrm{y}_{\mathrm{t}}-\mathrm{y}_{\mathrm{T}}\right)=0$. It will yield that $f_{x a}, f_{y a}$, and $v_{\text {apf }}$ equal to 0 . It means that the $v$ signal converges at the origin $(0,0)$. In every initial point, if the condition $\rho_{o}<c_{o b s}$ is achieved, then $v_{\text {apf }}=0$ because $\left(\mathrm{x}_{\mathrm{t}}-\mathrm{x}_{\mathrm{T}}\right)$ and $\left(\mathrm{y}_{\mathrm{t}}-\mathrm{y}_{\mathrm{T}}\right)$ must be have 0 of value. It can be concluded that the $v$ signal is stable.

\section{Proof 2 of preposition 2}

Since repulsive is not considered, variable $f_{x a}$ and $f_{y a}$ always be 0 . Therefore, $\operatorname{atan} 2(0 / 0)=0$ and variable $\alpha_{\text {apf }}$ depends on $\theta$. It means that $\beta_{\text {apf }}$ has 0 of value and $\omega=k_{\alpha} \theta$. Thus, angular velocity merely depends on $\theta$. When $k_{\alpha}$ is satisfied (41), then the system is stable.

\section{RESULT AND DISCUSSIONS}

In order to proof the concept, firstly, the test was conducted using Matlab via Simulink. The method can be depicted in the Simulink as seen as in Fig. 5.

From the Fig. 5, it can be seen that the result of $(x, y)$ of the APF is used as equilibrium point in the kinematic control. The passing value means that the trajectory of APF is used as reference equilibrium point of kinematic control.

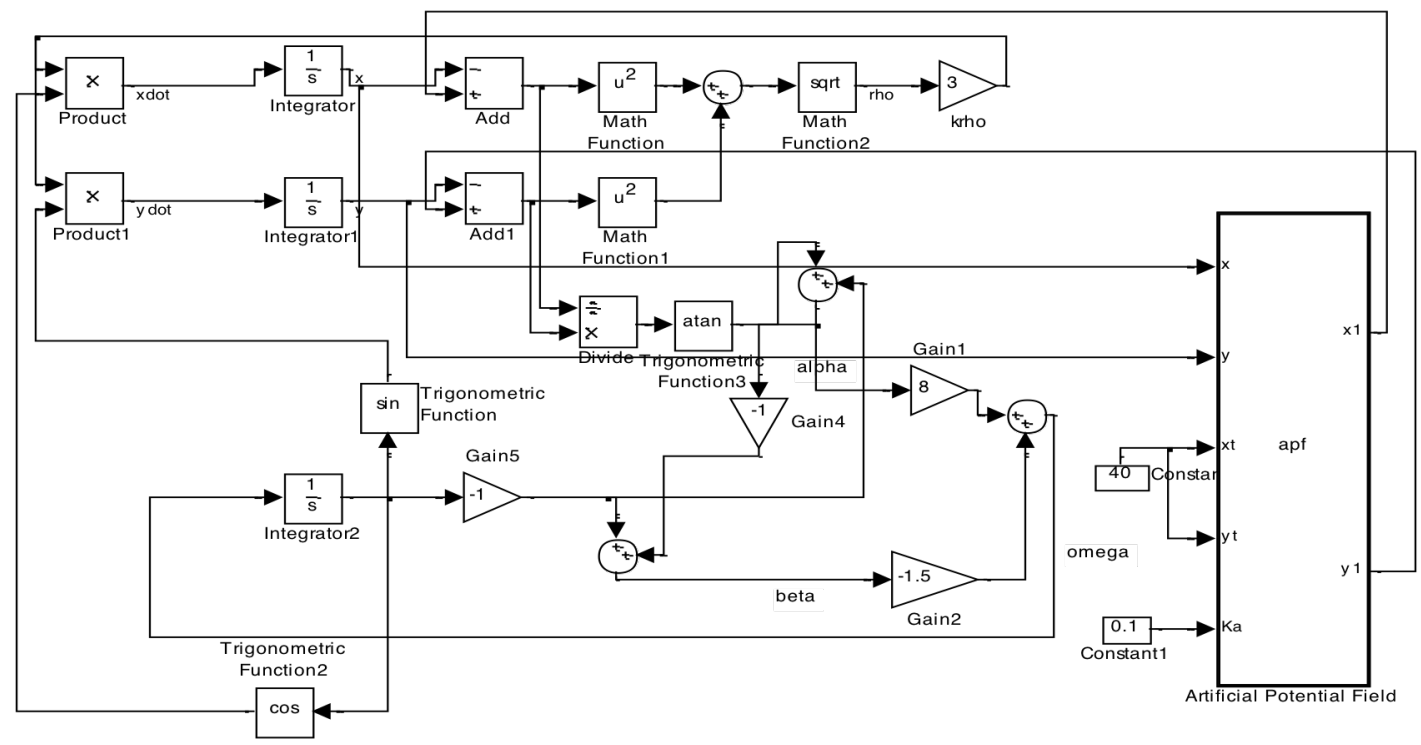

Fig. 5 Proposed Method via Matlab Simulink 


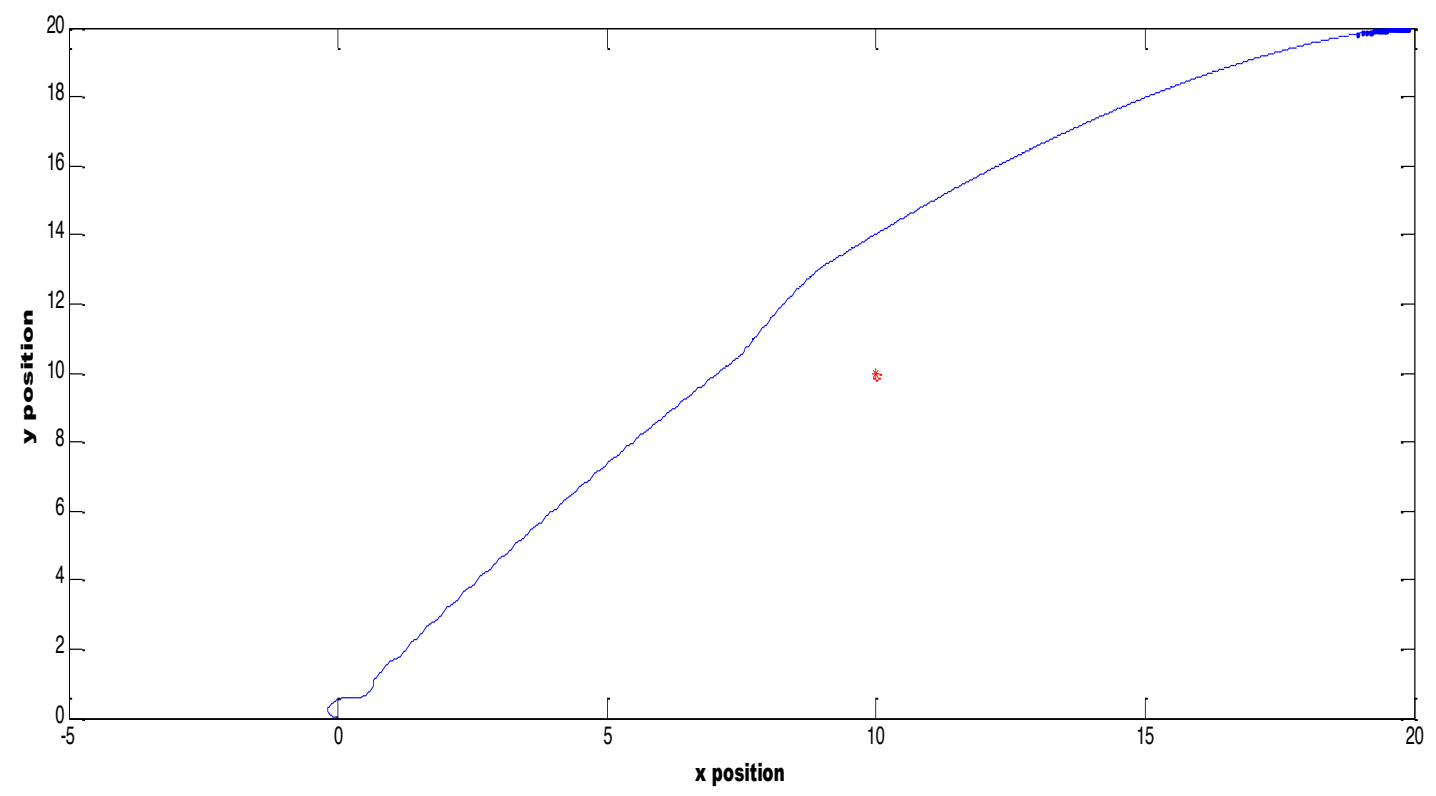

Fig. 6 Result of the proposed method (in 2D)

By setting the initial, goal, and the obstacles randomly, the integrated path planning with kinematic feedback control was running. The obstacle is assumed as point with certain radius. In the test, ode45 is used as the solver of the robot. The result can be depicted in the Fig. 6 .

From the Fig. 6, it can be shown that the robot start with different direction against the goal. The different angle is phi. The Fig. 6 shows that the control can compensate the nonholonomic constraint. The moving of the robot is not in linear movement although the goal position is in linear position. Curve movement is the compensation of the algorithm to the nonholonomic constraint. The benefit of the system is that can avoid the obstacle.

The second simulation was conducted on ROS (Robot Operating System). In the ROS, this research used Kobuki Yunjin robot as the platform which can be seen in Fig. 7.

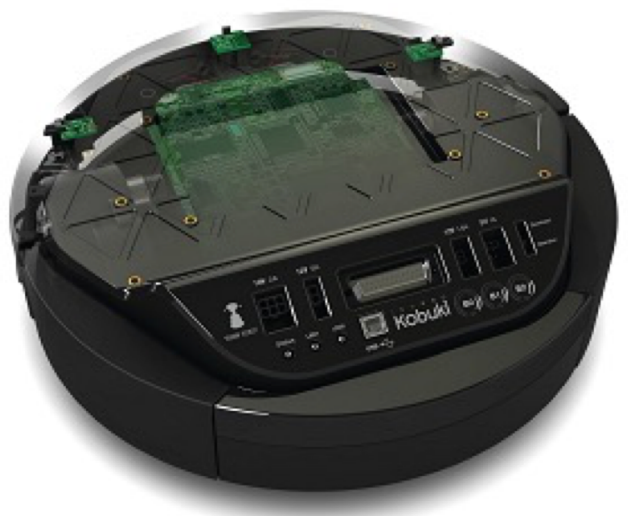

Fig. 7 Kobuki Yunjin Robot
The Kobuki robot has its characteristic of the orientation as seen in Table 1.

TABLE 1

TRANFORMATION OF THE KOBUKI ORIENTATION

\begin{tabular}{|c|c|}
\hline Kobuki & $\begin{array}{c}\text { Transfomation to } \\
\text { radian }\end{array}$ \\
\hline 1 & 0 \\
\hline 0 & $\pi$ \\
\hline-1 & $2 \pi$ \\
\hline
\end{tabular}

Regarding to the orientation characteristic, some rules must be define to adapt the artificial potential field control.

$$
\begin{gathered}
\text { if } \theta>0, \theta_{\text {conversion }}=(1-\theta)^{*} \pi, \\
\text { if } \theta<0, \theta_{\text {conversion }}=(1+\theta)^{*}(-\pi) .
\end{gathered}
$$

In the ROS simulation, the linear velocity of artificial potential field control can be implemented directly. Differently, in the angular velocity, the value must be controlled by specific gain. This research set the gain of 0.009 . Therefore, the formula of angular velocity is

$$
\omega_{\text {norm }}=0.009 * \omega .
$$

The scenario on the ROS simulation is that the Kobuki robot moved from its initial and has to avoid an obstacle in between the robot and its origin. The origin was set at $(0,0)$. The scenario of the proposed method can be depicted in Fig. 8 . 


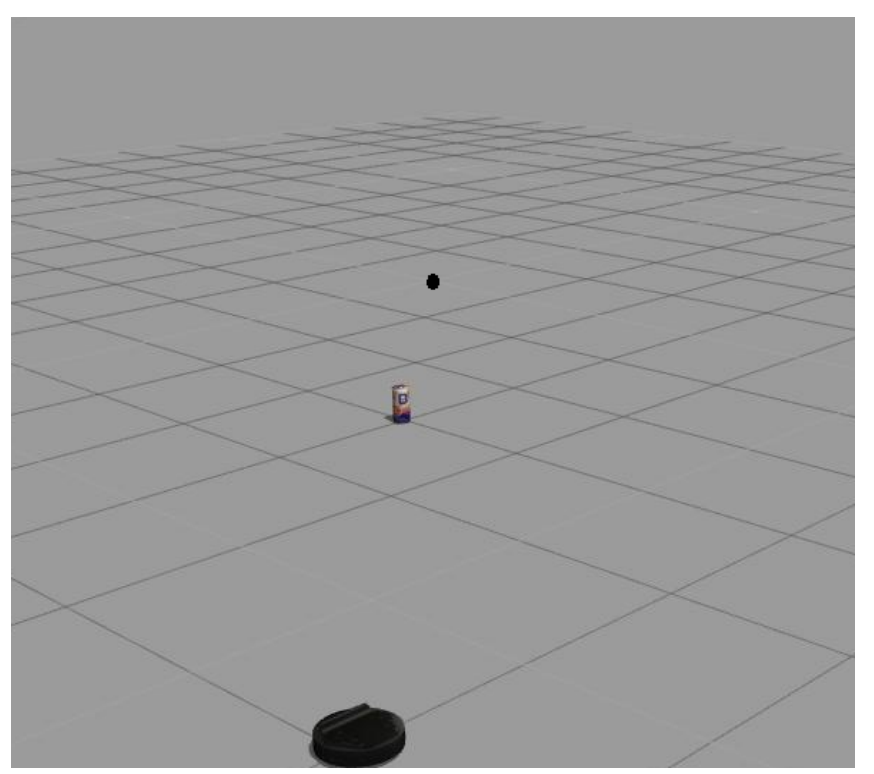

Fig. 8 Scenario of ROS simulation beer bottle - an obstacle, black dot - origin

The result can be seen in Fig. 9.

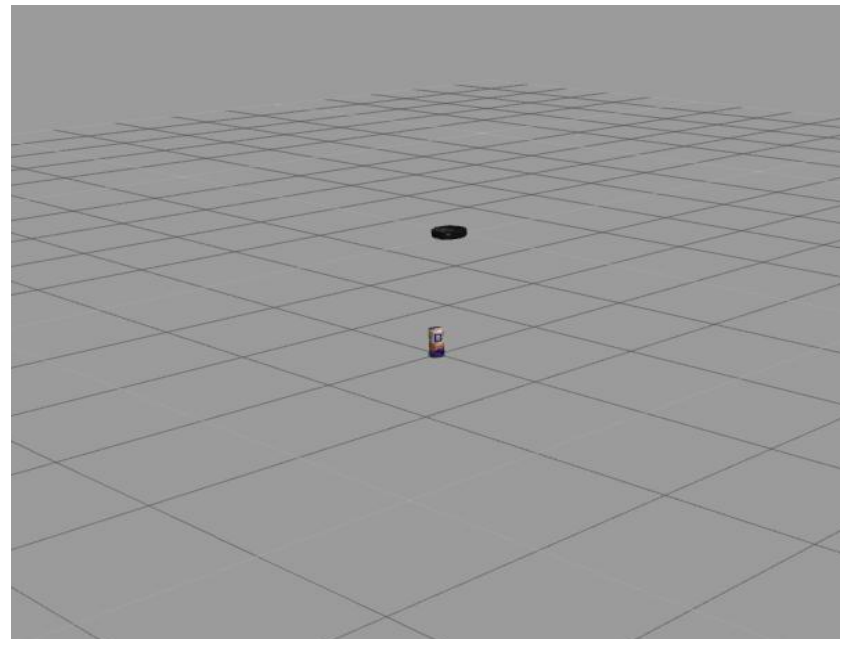

Fig. 9 Result the proposed method on the ROS simulation

The result of the ROS simulation was shown that the proposed method avoids the obstacle and convergent at the origin.

It has to be noted that the Kobuki used inertial frame as reference to calculate the distance. The odometry function was turn off because of the error measurement that will influence to performance of the proposed method.

Fig. 10 and 11 show the movement of the robot in $\mathrm{x}$ and $\mathrm{y}$ axis. From both of the Figures, the algorithm can meet convergent and reach the goal position. It means that the algorithm has been proven stable.

From the local stability analysis, it has been proven that the system is stable if the requirement of (40) has been fulfilled. The analysis of the force is divided into two conditions. The conditions are following the rule in (42). The condition when $\rho_{o}>c_{o b s}$ can be proven easily by using assumption the equilibrium or the goal point is $(0,0)$.

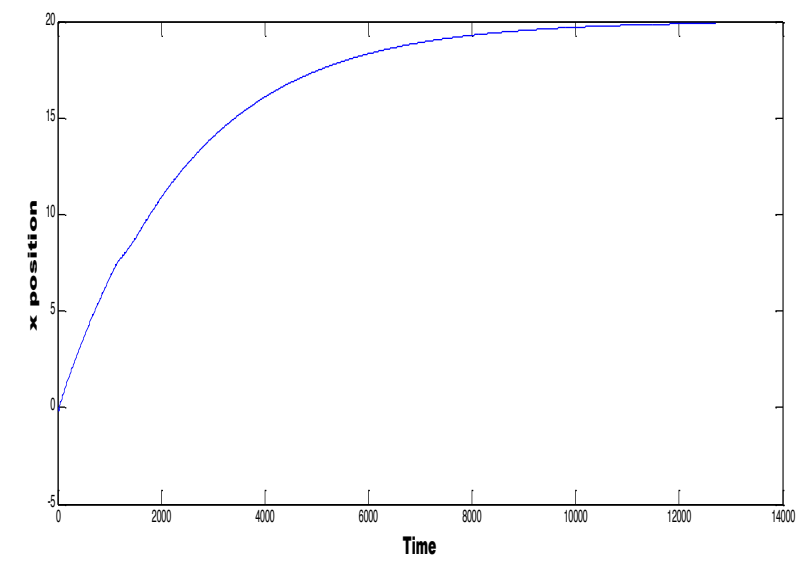

Fig. 10 Result of the proposed method (x position vs time)

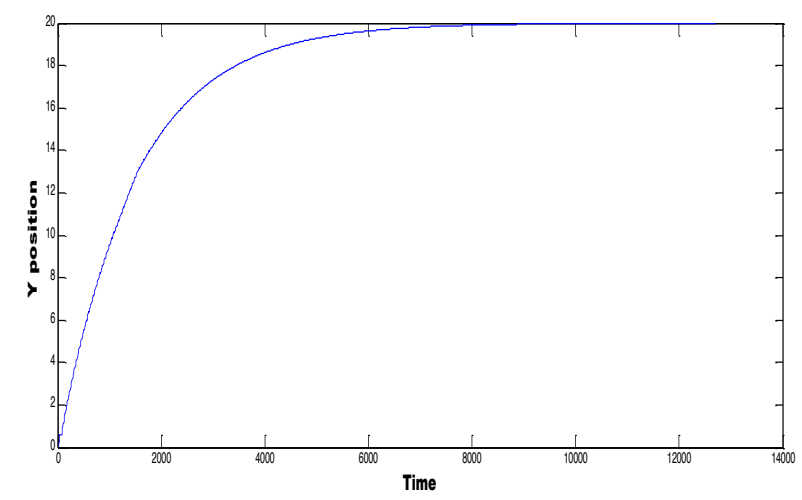

Fig. 11 Result of the proposed method (y position vs time)

\section{CONCLUSIONS}

The assumption of the robot as a point mass becomes one of the problems how to cope the nonholonomic constraint in APF. An Artificial Potential path planning with kinematic control has been delivered to handle the problem. A simple idea that was proposed is converting the equilibrium point on the kinematic control as the position on the APF. Thus, the states of the kinematic control become dynamic depending on the APF. On the other hand, the position of APF is determined by (18).

The control rules in the kinematic control used APF and PD (Proportional and Derivative Control). The magnitude of APF is applied on linear velocity control input. Considering the pose of APF that avoid the obstacle, the direction of APF is employed to control the angular velocity. The variable $\alpha$ is influenced by the direction of the APF and will impact to $\omega$. Consequently, the robot will move to the direction of the APF to go to the next APF point. The control is implemented based on two conditions, i.e. obstacle and non-obstacle environment.

By the combining of APF and the kinematic control, besides can solve nonholonomic constraint, the kinematic control can avoid the obstacle. This happens because the APF is employed as guidance trajectory of kinematic control. The result of the simulation shows that although the equilibrium point of the kinematic control is adapted to the APF but the control meet stability and convergent. 
In this research, the assumption of the localization is perfect. It means that the robot moves in the right direction and position without an error. Real time platform encounter noise error from the sensor and it will impact to the proposed method. Furthermore, real time implementation should be conducted to proof the robustness of the proposed algorithm.

\section{ACKNOWLEDGMENT}

This research was supported by PUPT Project grant from DIKTI through Research Directorate, Universitas Gadjah Mada with the contract number: 944/UN1-P.III/LT/DITLIT/2016, awarded to A/P Dr. Oyas Wahyunggoro.

\section{REFERENCES}

[1] T. Tomic, K. Schmid, P. Lutz, A. Domel, M. Kassecker, E. Mair, I. Grixa, F. Ruess, M. Suppa, and D. Burschka, "Toward a fully autonomous UAV: Research platform for indoor and outdoor urban search and rescue," IEEE Robot. Autom. Mag., vol. 19, no. 3, pp. 46$56,2012$.

[2] M. Shanmugavel, "Path Planning of Multiple Autonomous Vehicles," Cransfield University, 2007.

[3] M. R. Walter, "Sparse Bayesian Information Filters for Localization and Mapping," Massachusetts Institute of Technology, 2008.

[4] A. a. Masoud, "A harmonic potential field approach for joint planning and control of a rigid, separable nonholonomic, mobile robot," Rob. Auton. Syst., vol. 61, no. 6, pp. 593-615, 2013.

[5] J. M. Martín Ramos, D. López García, F. Gómez-Bravo, and a. Blanco Morón, "Application of multicriteria decision-making techniques to manoeuvre planning in nonholonomic robots," Expert Syst. Appl., vol. 37, no. 5, pp. 3962-3976, 2010.

[6] Y. V. Pehlivanoglu, "A new vibrational genetic algorithm enhanced with a Voronoi diagram for path planning of autonomous UAV," Aerosp. Sci. Technol., vol. 16, no. 1, pp. 47-55, 2012.

[7] A. Tuncer and M. Yildirim, "Dynamic path planning of mobile robots with improved genetic algorithm," Comput. Electr. Eng., vol. 38, no. 6, pp. 1564-1572, 2012.

[8] P. S. Xuan Zou, Bin Ge, "Improved Genetic Algorithm for Dynamic Path Planning," Int. J. Inf. Comput. Sci. IJICS, vol. 1, no. 2, pp. 16$20,2012$.

[9] O. Montiel, U. Orozco-Rosas, and R. Sepúlveda, "Path planning for mobile robots using Bacterial Potential Field for avoiding static and dynamic obstacles," Expert Syst. Appl., vol. 42, no. 12, pp. 5177-5191, 2015

[10] M. A. Hossain and I. Ferdous, "Autonomous robot path planning in dynamic environment using a new optimization technique inspired by bacterial foraging technique," Rob. Auton. Syst., vol. 64, pp. 137-141, 2015.

[11] J.-H. Liang and C.-H. Lee, "Efficient collision-free path-planning of multiple mobile robots system using efficient artificial bee colony algorithm," Adv. Eng. Softw., vol. 79, pp. 47-56, 2015.

[12] G. Wang, Q. Li, and L. Guo, "Multiple UAVs Routes Planning Based on Particle Swarm Optimization Algorithm," 2010 2nd Int. Symp. Inf. Eng. Electron. Commer., pp. 1-5, 2010.

[13] J. Karimi and S. H. Pourtakdoust, "Optimal maneuver-based motion planning over terrain and threats using a dynamic hybrid PSO algorithm," Aerosp. Sci. Technol., vol. 26, no. 1, pp. 60-71, 2013.

[14] Y. Liu, M. Li, C. Xie, M. Peng, and F. Xie, "Path-planning research in radioactive environment based on particle swarm algorithm," Prog. Nucl. Energy, vol. 74, pp. 184-192, 2014.

[15] H. Mo and L. Xu, "Neurocomputing Research of biogeography particle swarm optimization for robot path planning," Neurocomputing, vol. 148, pp. 91-99, 2015.

[16] S. Chakraborty, "Ant Colony System : A New Concept to Robot Path Planning,” Int. J. Hybrid Inf. Technol., vol. 6, no. 6, pp. 11-30, 2013.
[17] W. Zhangqi, Z. Xiaoguang, and H. Qingyao, "Mobile Robot Path Planning based on Parameter Optimization Ant Colony Algorithm," Procedia Eng., vol. 15, pp. 2738-2741, 2011.

[18] M. A., V. Ayala-Ramirez, and U. H. Hernandez-Belmonte, "Mobile robot path planning using artificial bee colony and evolutionary programming," Inf. Sci. (Ny)., vol. 238, pp. 319-328, 2015.

[19] E. Besada-Portas, L. De La Torre, A. Moreno, and J. L. Risco-Martin, "On the performance comparison of multi-objective evolutionary UAV path planners," Inf. Sci. (Ny)., vol. 238, pp. 111-125, 2013.

[20] A. Sgorbissa and R. Zaccaria, "Planning and obstacle avoidance in mobile robotics," Rob. Auton. Syst., vol. 60, no. 4, pp. 628-638, 2012

[21] M. Shanmugavel, A. Tsourdos, B. White, and R. Zbikowski, "Cooperative path planning of multiple UAVs using Dubins paths with clothoid arcs," Control Eng. Pract., vol. 18, no. 9, pp. 1084-1092, 2010.

[22] M. Shanmugavel, A. Tsourdos, and B. A. White, "Collision avoidance and path planning of multiple UAVs using flyable paths in 3D," in 2010 15th International Conference on Methods and Models in Automation and Robotics, MMAR 2010, 2010, pp. 218-222.

[23] M. Elbanhawi, M. Simic, and R. Jazar, "Randomized Bidirectional BSpline Parameterization Motion Planning,” IEEE Trans. Intell. Transp. Syst., vol. 17, no. 2, pp. 406-419, 2016.

[24] H. H. Triharminto, A. S. Prabuwono, T. B. Adji, and N. A. Setiawan, "Adaptive Dynamic Path Planning Algorithm for Interception of a Moving Target," Int. J. Mob. Comput. Multimed. Commun., vol. 5, no. 3, pp. 19-33, Jul. 2013.

[25] C. Y. C. Yong and E. J. Barth, "Real-time Dynamic Path Planning for Dubins' Nonholonomic Robot," Proc. 45th IEEE Conf. Decis. Control, pp. 2418-2423, 2006.

[26] A. S. Matveev, H. Teimoori, and A. V. Savkin, "Navigation of a unicycle-like mobile robot for environmental extremum seeking," Automatica, vol. 47, no. 1, pp. 85-91, 2011.

[27] J. Cochran, S. Member, and M. Krstic, "Nonholonomic Source Seeking With Tuning of Angular Velocity," IEEE Trans. Automat. Contr., vol. 54, no. 4, pp. 717-731, 2009.

[28] M. Kal'avský and Ž. Ferková, "Harmonic Potential Field Method for Path Planning of Mobile Robot," in International Virtual Conference 2012 (ICTIC 2012) Slovakia, 2012, no. 1, pp. 41-46.

[29] M. Michałek and K. Kozłowski, "Vector-field-orientation feedback control method for a differentially driven vehicle," IEEE Trans. Control Syst. Technol., vol. 18, no. 1, pp. 45-65, 2010.

[30] L. Xie, H. Chen, and G. Xie, "Artificial Potential Field Based Path Planning for Mobile Robots Using Virtual Water-Flow Method," in Third International Conference on Intelligent Computing, 2007, pp. 588-595.

[31] Z. Zeng, L. Lian, K. Sammut, F. He, Y. Tang, and A. Lammas, “A survey on path planning for persistent autonomy of autonomous underwater vehicles," Ocean Eng., vol. 110, pp. 303-313, 2015.

[32] S. Hassan and J. Yoon, "Haptic assisted aircraft optimal assembly path planning scheme based on swarming and artificial potential field approach," Adv. Eng. Softw., vol. 69, pp. 18-25, 2014.

[33] M. K. Weir and M. P. Bott, "High quality goal connection for nonholonomic obstacle navigation allowing for drift using dynamic potential fields," in 2010 IEEE International Conference on Robotics and Automation, 2010, pp. 3221-3226.

[34] A. A. Ahmed, T. Y. Abdalla, and A. A. Abed, "Path Planning of Mobile Robot by using Modified Optimized Potential Field Method," Int. J. Comput. Appl., vol. 113, no. 4, pp. 6-10, 2015.

[35] T. Urakubo, "Feedback stabilization of a nonholonomic system with potential fields: application to a two-wheeled mobile robot among obstacles," Nonlinear Dyn., vol. 81, no. 3, pp. 1475-1487, Apr. 2015.

[36] T. Urakubo, K. Okuma, and Y. Tada, "Feedback control of a two wheeled mobile robot with obstacle avoidance using potentia functions," 2004 IEEE/RSJ Int. Conf. Intell. Robot. Syst. (IEEE Cat. No.04CH37566), vol. 3, pp. 2428-2433, 2004.

[37] R. Siegwart and I. R. Nourbakhsh, Introduction to Autonomous Mobile Robots, vol. 23. 2004.

[38] O. Khatib, "Real-Time Obstacle Avoidance for Manipulators and Mobile Robots," pp. 500-505, 1985 